\title{
Colorectal cancer patient's self-efficacy for managing illness-related problems in the first 2 years after diagnosis, results from the ColoREctal Well-being (CREW) study
}

\author{
Chloe Grimmett $^{1}$ - Joanne Haviland ${ }^{2}$. Jane Winter ${ }^{1,3} \cdot$ Lynn Calman $^{1} \cdot$ Amy Din $^{1}$ • \\ Alison Richardson ${ }^{3,4}$ - Peter W. F. Smith ${ }^{5}$. Claire Foster ${ }^{1}$
}

Received: 24 March 2017 / Accepted: 1 August 2017 /Published online: 19 August 2017

(C) The Author(s) 2017. This article is an open access publication

\begin{abstract}
Purpose There is a growing emphasis on self-management of cancer aftercare. Little is known about patient's self-efficacy (confidence) to manage illness-related problems and how this changes over time. This paper describes the patterns of selfefficacy for managing illness-related problems amongst colorectal cancer patients in the 2 years following diagnosis.

Methods In this prospective cohort study, questionnaires were administered at baseline (pre-surgery), 3, 9, 15 and 24 months to 872 colorectal cancer patients. Self-efficacy (confidence to manage illness-related problems), anxiety, social support, affect, socio-demographics, physical symptoms and clinical and treatment characteristics were assessed. Group-based trajectory analysis identified trajectories of self-efficacy up to 24 months and predictors.

Results Four trajectories of self-efficacy were identified: group 1 (very confident) $16.0 \%$ (95\% confidence interval (CI) $10.7-21.3 \%$ ), group 2 (confident) $45.6 \%$ (95\% CI $40.3-51.0 \%$ ), group 3 (moderately confident) $29.5 \%$ (95\% CI 25.1-33.8\%) and group 4 (low confidence) $8.9 \%$ (95\%
\end{abstract}

Claire Foster

C.L.Foster@soton.ac.uk

1 Macmillan Survivorship Research Group, Faculty of Health Sciences, University of Southampton, Southampton SO17 1BJ, UK

2 The Institute of Cancer Research, 15 Cotswold Road, Sutton, London SW7 3RP, UK

3 University Hospital Southampton NHS Foundation Trust, Southampton SO16 6YD, UK

4 Faculty of Health Sciences, University of Southampton, Southampton SO17 1BJ, UK

5 Social Statistics and Demography, Social Sciences, University of Southampton, Southampton SO17 1BJ, UK
CI 6.4-11.4\%). Greater deprivation, domestic status, more co-morbidities, worse fatigue and pain, lower positivity and greater negativity were significantly associated with lower self-efficacy. There was an increase in mean scores for selfefficacy over time for the whole sample, but this did not reach the cut-off for minimally important differences. At 2 years, the lowest level of confidence to manage was for symptoms or health problems.

Conclusion Around $40 \%$ of patients had suboptimal levels of confidence to manage illness-related problems with little change from the time of diagnosis across the four groups. Implications for cancer survivors Screening for self-efficacy at diagnosis would enable targeted, early intervention which could in turn enhance health-related quality of life.

Keywords Colorectal · Cancer · Self-efficacy ·

Self-management

\section{Introduction}

Cancer is increasingly being viewed as a chronic health condition with evidence that cancer and its treatment can have a significant impact on people's lives in the months and years following treatment [1-3]. This is reflected in a growing emphasis on selfmanagement of cancer aftercare. Here, the patient is expected to manage the physical and psychosocial consequences of cancer and its treatment, seeking support from the health care system when they feel it necessary, and making lifestyle changes where appropriate to improve health and well-being.

Identifying factors associated with a person's capability to self-manage after cancer can enhance understanding of how and when to best support them. Self-efficacy, defined by Bandura as 'beliefs in one's capabilities to organize and execute 
the courses of action required to manage prospective situations' [4], has been identified as a personal resource which may promote adjustment to cancer and other chronic illnesses and is amendable to change.

Data from cross-sectional studies support a positive association between self-efficacy and quality of life [5], adjustment following a cancer diagnosis [6, 7] and symptom distress [8]. Self-efficacy to manage illness-related problems may also moderate the relationship between a physical symptom and quality of life. In a study of 112 women receiving adjuvant endocrine therapy, increasing physical symptoms were only associated with lower functional and emotional well-being in those reporting low self-efficacy for coping with symptoms [9].

There are limited data describing if and how self-efficacy changes over time in cancer populations. Knowledge of patterns of change in self-efficacy from diagnosis and in the years following could highlight the best time to intervene to support those who might be struggling. Existing longitudinal studies tend to focus on breast cancer or mixed cancer populations, with limited data available in colorectal cancer patients. They also have methodological limitations, considerable variability in sample characteristics, and unsurprisingly, reach conflicting conclusions.

Conflicting data exists as to the stability of self-efficacy over time in cancer populations. In one of the earliest studies, Lev and colleagues [10] followed a cohort of 307 cancer patients for 8 months and concluded that mean self-care selfefficacy reduced over time. However, the sample included patients with metastatic disease and less than half completed the 8-month follow-up assessment. In contrast, Manne et al. [5] and Rottman et al. [11] describe cancer-related self-efficacy and general self-efficacy, respectively, remained stable over a 12 -month period. However, in a sample of 95 breast cancer patients over a period of 12 months, the exception was the subscales of self-efficacy for activity management and selfsatisfaction increased [5]. This study employed the Stanford Inventory of Cancer Patient Adjustment (SICPA) scale. The authors acknowledge that, unlike the other subscales, for the two facets of self-efficacy which improved, respondents were asked to assess confidence in their abilities to manage activities compared to before their diagnosis. This may account for the difference. It should also be noted that the psychometric properties of SICPA have not been confirmed.

Finally, in a more recent study, Mystakidou and colleagues followed 90 cancer patients receiving radiotherapy, assessing their general perceived self-efficacy before radiotherapy commenced and 1 month after completion of their treatment [12]. They recount self-efficacy levels reduced during this time, and were accompanied by an increase in anxiety and deterioration in quality of life. However, the sample size was small, followup short and cancer type varied, limiting generalisability.

As well as methodological concerns and sample heterogeneity of the aforementioned studies, they also vary considerably regarding the definition and measure of self-efficacy used. This includes instruments assessing perceived general self-efficacy (e.g. the General Self-Efficacy scale [13]) to more specific measures of self-efficacy to cope with cancer-related problems, such as the Cancer Behavior Inventory [14]. We argue, in line with Bandura's definition, that self-efficacy is a situation-specific competence belief [15], and as others have said [9] that the specificity of the measures used in this context is of utmost importance. Previous data demonstrate that even within a focussed measure of self-efficacy, the Self-Efficacy for Managing Chronic Disease scale [16], there is substantial variation in patient's perceived ability to manage varying aspects of their illness. For example, self-efficacy to manage fatigue was significantly lower than self-efficacy to access information in a cross-sectional sample of cancer survivors within 1 year of cancer treatment [17]. If the most pertinent targets for intervention are to be determined in the changing landscape of selfmanagement support after cancer treatment, we need to first understand the specific areas of disease self-management that require most support, who is most likely to need support and when.

Previous literature is limited by the reporting of mean selfefficacy scores for the populations under investigation, potentially masking groups of patients with higher or lower selfefficacy. Recently, trajectories of psychosocial and quality of life outcomes have been reported. These analyses determine whether there are distinct groups within the sample, i.e. those scoring consistently low/high during the period of follow-up, which would be masked by group mean scores. In a prospective cohort of colorectal cancer survivors followed from 5 months to 5 years post-diagnosis, Dunn et al. [18] report four trajectories of psychological distress with $19 \%$ of the sample reporting consistently low levels of distress. This analytical method was also used by our research group to examine trajectories of quality of life and well-being in the CREW cohort: a large representative group of colorectal cancer patients being treated with curative intent from the point of diagnosis at regular intervals for 2 years. Using the Quality of Life in Adult Cancer Survivors (QLACS) inventory to measure quality of life, we found four distinct trajectories of recovery with two groups faring consistently well (above the median), and two groups (below the median) who consistently struggled [19].

The current study aims to overcome some of the limitations of the previous literature, reporting data from the CREW cohort study. In addition to a prospective longitudinal study design with a pre-surgical baseline assessment, we use a measure of self-efficacy specific to the management of chronic illness: the Self-Efficacy for Managing Chronic Disease scale [16]. The objectives of this paper are to describe patterns of self-efficacy for managing illness-related problems using trajectory analysis, identify areas where self-efficacy is lowest and examine the baseline predictors of self-efficacy in the first 2 years since diagnosis. 


\section{Methods}

\section{Design}

CREW is a prospective, longitudinal cohort study with patients recruited from 29 UK hospitals prior to surgery with curative intent colorectal cancer. Full details of the CREW study protocol can be found elsewhere [20].

\section{Participants}

Eligibility criteria included (a) a diagnosis of colorectal cancer (Dukes A-C), (b) awaiting primary surgery with curative intent, (c) $\geq 18$ years old and (d) had the ability to complete questionnaires (language line translation facilities were available for those that did not speak English). Exclusions included distant metastatic disease at diagnoses or a prior diagnosis of cancer (other than non-melanomatous skin cancer or in situ carcinoma cervix).

\section{Procedure}

Written informed consent was obtained and the UK National Health Service National Research Ethics Service (REC reference number 10/H0605/31) approved conduct of the study. Eligible patients were identified by a member of their clinical care team during multidisciplinary team meetings at one of the 29 UK cancer centres involved in CREW. Baseline questionnaires were completed prior to primary surgery wherever possible with subsequent follow-up at 3, 9, 15 and 24 months post-surgery and annual assessments are ongoing. Clinical and treatment details were gathered (with consent) from medical notes. This included stage, type and grade of disease as well as details of type of surgery, chemotherapy and radiotherapy. Postcode was used to derive the Index of Multiple Deprivation, representing neighbourhood deprivation.

\section{Measures}

Areas of assessment were informed by our recovery framework describing constructs believed to be important in recovery from cancer diagnosis (see [21] for full details). Validated measures were repeated at every time point unless otherwise indicated.

Self-efficacy was assessed at all time points using the Selfefficacy for Managing Chronic Disease (SEMCD) scale [16]. From baseline to 9 months inclusive, the original six-item version of the scale was used; from 15 months onwards, an additional five cancer-specific items were included, comprising the Cancer Survivors' Self-Efficacy Scale (CS-SES [22]). The CE-SES was found to have excellent reliability (Cronbach's alpha 0.92) with the 11-item scale) [22]. At 2 years, the term 'your disease' was changed to 'your cancer' throughout this measure to ensure respondents were relating the items to their cancer experience rather than other co-morbid conditions. For example, the item 'How confident are you that you can keep the emotional distress caused by your disease from interfering with the things you want to do?' was changed to 'How confident are you that you can keep the emotional distress caused by your cancer from interfering with the things you want to do?' All items are measured on a scale from 1 (not at all confident) to 10 (totally confident), and an overall score is obtained by taking the mean of all the individual items.

State anxiety was measured using the State-Trait Anxiety Inventory (STAI [23]) consisting of 20 items. Higher scores indicate greater anxiety. Scores $\geq 40$ suggest clinically significant anxiety.

Depression was assessed using the 20-item Centre for Epidemiological Studies Depression (CES-D [24]) scale. Higher scores indicate greater depression and scores of $\geq 20$ suggest clinical depression [25].

The Medical Outcomes Study (MOS) measured social support. The scale includes 19 items, 18 of which comprise four subscales: emotional/informational, tangible, affectionate support and positive social interaction. Higher scores denote greater social support [26].

Symptoms of pain and fatigue were examined using the subdomains of the QLACS scale, each subdomain consisting of four items. Higher scores indicate worse pain and fatigue [27]. These symptoms were highly prevalent and two of the items of the SEMCD scale refer specifically to pain and fatigue.

Positive and negative affect was assessed by the Positive and Negative Affect Schedule (PANAS) Short Form [28]. Two five-item mood scales measure positive and negative affects with higher scores depicting stronger positive or negative emotions.

\section{Statistical methods}

Published guidance for dealing with missing items in subscales were applied where available; otherwise, providing at least $75 \%$ of individual items within a subscale had been completed. The scores of any missing items within the scale were taken to be the mean of the scores of the available items. Participants with missing questionnaires, including baseline, were included in analyses for time points for which they provided data, and there was no imputation of missing questionnaires from other completed questionnaires. The Index of Multiple Deprivation was categorised into quintiles.

Cross-sectional analyses summarised mean scores for the overall self-efficacy scales (6- and 11-item versions), as well as individual items from baseline to 2 years following surgery. Changes in the overall mean score and individual items of the six-item scale from baseline to 2-year follow-up of at least half a standard deviation (SD) were assessed as minimally important differences [29].

Group-based trajectory analyses [30] were used to investigate whether distinct groups could be identified for the six-item 
SEMCD scale from baseline to 24 months. These are discrete mixture models, which modelled the SEMCD score as censored normal data following a polynomial time curve. The optimal number of distinct trajectories was determined using the Bayesian information criterion (BIC) [31,32] to compare model fit (a change in BIC $>10$ supports the more complex model), whilst aiming to avoid trajectories containing very few individuals. The shape of each trajectory was assessed to determine whether it was best described by a linear, quadratic or cubic function according to the significance of each term. Estimated proportions of participants within each trajectory were obtained from the models, with $95 \%$ CIs. Potential predictors of trajectory group membership were included in the models within the following domains: (a) socio-demographic factors at baseline (age, gender, domestic status, neighbourhood deprivation), (b) clinical and treatment factors (tumour site, Dukes stage, co-morbidities, neoadjuvant and adjuvant treatment, stoma), (c) physical symptoms at baseline (fatigue and pain) and (d) psychosocial factors at baseline (positive and negative affects, anxiety, depression, social support). All factors found to be statistically significant $(p<0.05)$ for at least one of the trajectories were then included together in an overall model to find which factors remained independent significant predictors of group membership. Statistical significance of model parameters was assessed by the Wald test. For predictors of trajectory group membership, the following were included as continuous variables in the model: age, fatigue, pain, positive and negative affects, overall social support and the categorical data fitted as factors: gender, domestic status, neighbourhood deprivation, clinical and treatment factors, anxiety and depression.

Date of surgery was taken as time zero and follow-up time calculated using date of questionnaire completion; timing of baseline questionnaire (pre-/post-surgery) was adjusted for in all trajectory models as approximately $30 \%$ of participants completed their baseline after surgery due to logistical reasons, including emergency surgery. To ease interpretation of the estimated trajectories, the following cut-offs were assigned to the six-item SEMCD scale: 1-4 'low confidence', 5-6 'moderate confidence', 7-8 'confident' and 9-10 'very confident'.

\section{Results}

\section{Study participants}

A total of 857 participants consented to follow-up (excluding 15 who withdrew at baseline). Response rates of those remaining eligible for questionnaires at each time point were $88 \%$ at baseline, $84 \%$ at 3 months, $82 \%$ at 9 months, $80 \%$ at 15 months and $74 \%$ at 24 months at the point of analysis. Full details of participants are described elsewhere [19]. In brief, the sample comprised $65 \%$ colon and $35 \%$ rectal cancer patients; disease stage was 14\% Duke's A, 53\% Duke's B and
$32 \%$ Duke's C; $18 \%$ received neoadjuvant and $35 \%$ adjuvant therapy; and $35 \%$ had a stoma (most temporary). The mean age of participants was 68 years, with $60 \%$ male. By 2 years, $79(9.9 \%)$ had experienced a recurrence, $65(7.6 \%)$ had died and $105(12.2 \%)$ had withdrawn.

\section{Levels of self-efficacy over follow-up}

Self-efficacy scores (overall mean scores and for individual items of 6-item SEMCD scale and 11-item CS-SES scale) are shown in Table 1. For the group as a whole, there was evidence of an increase in self-efficacy over the 2-year follow-up, with statistically significant changes in the overall mean score $(p<0.001)$ and some individual scores $(p<0.01$ for all subscores on the sixitem scale), although the absolute change in scores was small. The mean score of the six-item SEMCD scale increased from 7.4 $(\mathrm{SD}=1.9)$ at baseline to $8.2(\mathrm{SD}=1.8)$ at 2 years, not quite reaching the cut-off for a minimally important difference (MID; $0.5 \times \mathrm{SD})$.

At baseline, participants reported lowest levels of confidence with fatigue and physical discomfort/pain, and highest level for 'doing things other than just taking medication'. These improved over the 2 years in the group as a whole. At 2 years, the lowest level of confidence to manage was for symptoms or health problems and the highest for contacting the doctor. Comparing scores between baseline and 24 months for individual items, the following reached the above definition of a MID: physical discomfort/pain and emotional distress, with a borderline change for fatigue (Table 1).

The optimal number of distinct trajectories (groups) identified for the six-item SEMCD scale from surgery to 2 years was 4 (change in BIC from three groups to $4=165.57$ and change in BIC from four groups to $5=9.73$ ). Estimated proportions of the CREW sample in each group were $16.0 \%$ (95\% CI $10.7-21.3 \%$ ) in group 1 (very confident, with highest levels of self-efficacy in CREW), 45.6\% (95\% CI 40.3-51.0\%) in group 2 (confident), 29.5\% (95\% CI 25.1-33.8\%) in group 3 (moderate confidence) and $8.9 \%$ (95\% CI 6.4-11.4\%) in group 4 (low confidence, with lowest levels of self-efficacy) (Fig. 1). The small increase in levels of self-efficacy over follow-up found in the whole group was less apparent when examining the trajectories (Fig. 1), suggesting no clinically significant improvements over the 2-year follow-up.

\section{Associations with self-efficacy}

From the trajectory models including potential predictors of self-efficacy, the following baseline factors were found to be significantly associated with lower levels of self-efficacy over the 2-year follow-up: greater neighbourhood deprivation, domestic status (being single/widowed/divorced/separated), a greater number of co-morbidities, worse symptoms of fatigue and pain, lower positivity and greater negativity (Table 2). 
Table 1 Descriptive results for self-efficacy from baseline to 2 years following surgery

\begin{tabular}{|c|c|c|c|c|c|}
\hline $\begin{array}{l}\text { Mean (SD) shown; range is } 1-10 \text { for } \\
\text { all scales and individual items }\end{array}$ & $\begin{array}{l}\text { Baseline } \\
(N=756)\end{array}$ & $\begin{array}{l}3 \text { months } \\
(N=668)\end{array}$ & $\begin{array}{l}9 \text { months } \\
(N=623)\end{array}$ & $\begin{array}{l}15 \text { months } \\
(N=579)\end{array}$ & $\begin{array}{l}24 \text { months } \\
(N=514)\end{array}$ \\
\hline 6-item SEMCD scale & $7.4(1.9)$ & $7.6(2.0)$ & $7.8(1.9)$ & $8.0(1.8)$ & $8.2(1.8)$ \\
\hline 11-item CS-SES scale & N/A & N/A & N/A & $8.2(1.7)$ & $8.3(1.6)$ \\
\hline \multicolumn{6}{|l|}{ Self-efficacy (confidence) to manage } \\
\hline Fatigue & $7.2(2.2)$ & $7.3(2.3)$ & $7.5(2.2)$ & 7.9 & $8.1(2.1)$ \\
\hline Physical discomfort or pain & $7.2(2.1)$ & $7.6(2.2)$ & $7.7(2.2)$ & $8.1(2.0)$ & $8.4(1.9)$ \\
\hline Emotional distress & $7.3(2.2)$ & $7.7(2.2)$ & $7.9(2.1)$ & $8.2(2.0)$ & $8.4(1.9)$ \\
\hline Symptoms or health problems & $7.4(2.2)$ & $7.4(2.2)$ & $7.5(2.2)$ & $7.8(2.1)$ & $7.7(2.2)$ \\
\hline Different tasks and activities & $7.7(2.0)$ & $7.9(2.0)$ & $8.0(2.0)$ & $8.2(1.9)$ & $8.2(2.0)$ \\
\hline $\begin{array}{l}\text { Do things other than just take } \\
\text { medication }\end{array}$ & $7.8(2.1)$ & $7.8(2.1)$ & $8.0(2.1)$ & $8.1(2.0)$ & $8.2(2.0)$ \\
\hline Access information & N/A & N/A & N/A & $8.3(2.0)$ & $8.5(1.9)$ \\
\hline Access people to help and support & N/A & N/A & N/A & $8.4(2.0)$ & $8.5(1.9)$ \\
\hline Deal with problems by yourself & N/A & N/A & N/A & $8.0(2.1)$ & $8.1(2.0)$ \\
\hline Contact doctor & N/A & N/A & N/A & $8.5(2.0)$ & $8.6(2.0)$ \\
\hline Get support with problems & N/A & N/A & N/A & $8.2(2.1)$ & $8.3(2.1)$ \\
\hline
\end{tabular}

$N$ indicates the number of questionnaires returned at each time point; the completeness of data within questionnaires varies; e.g. the overall mean score for the six-item SEMCD/CS-SES scales was available for 741 participants at baseline, 656 at 3 months, 610 at 9 months, 568 at 15 months and 505 at 24 months. The extent of missing data on individual items ranged from $0.8 \%$ for the item 'how confident are you that you can keep the physical discomfort or pain of having had cancer and/or cancer treatment from interfering with the things you want to do?' at 24 months to $8.1 \%$ for the item 'How confident are you that you can deal by yourself with the problems cancer and/or cancer treatment has caused?' at 15 months

$S D$ standard deviation, SEMCD Self-efficacy in Managing Chronic Disease, 6 items original Lorig scale (measured at all time points), 11 items original six-item Lorig scale plus five additional cancer-specific items (measured at 15 and 24 months), N/A not available
Those who had a stoma also reported lower levels of selfefficacy. These characteristics showed statistically significant differences for at least one of the lower self-efficacy groups (groups 2,3,4) compared with the reference trajectory (group $1=$ very confident $)$ in the multiple regression model. For the

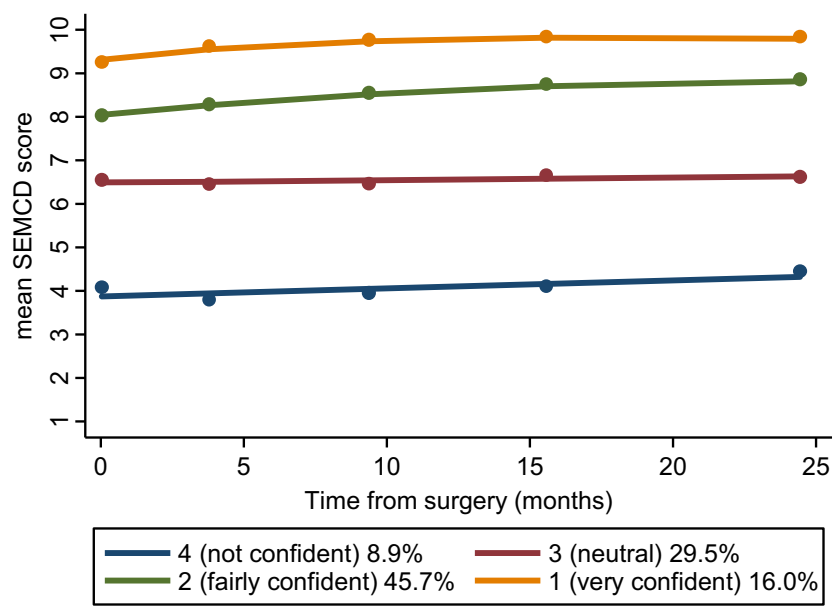

Fig. 1 Estimated trajectories of mean six-item SEMCD score from baseline to 24 months after surgery for 802 CREW participants with self-efficacy data comparison of group 4 (lowest self-efficacy) versus group 1 (highest self-efficacy), all of the listed participant characteristics were highly statistically significant $(p<0.01)$ except for neighbourhood deprivation (Table 2). There were no significant associations with self-efficacy group for age, gender, tumour site, Dukes stage, neoadjuvant and adjuvant treatment, anxiety, depression and social support.

\section{Discussion}

This paper reveals for the first time that across group trajectories, levels of self-efficacy remain stable in the first 2 years following colorectal cancer surgery with curative intent (pre-surgery and 3, 9, 12 and 24 months later). Sixty-two percent of the participants reported feeling very/fairly confident to manage their illnessrelated problems in the first 2 years. A third of the population reported feeling neither confident nor lacking in confidence, and the remaining 9\% lacked confidence to manage illness-related problems over the 2 years. This suggests that around $40 \%$ of those treated with curative intent for colorectal cancer could benefit from the offer of additional self-management support to manage illness-related problems. 
Table 2 Associations between baseline participant characteristics and level of self-efficacy (confidence to self-manage aspects of illness) up to 2 years after surgery: results from final trajectory model

\begin{tabular}{|c|c|c|c|c|c|}
\hline \multirow[t]{2}{*}{ Domain } & \multirow[t]{2}{*}{ Baseline characteristic } & \multicolumn{4}{|c|}{ Trajectory of six-item SEMCD scale } \\
\hline & & $\begin{array}{l}\text { Group 1: very } \\
\text { confident }(N=116)\end{array}$ & $\begin{array}{l}\text { Group 2: fairly } \\
\text { confident }(N=388)\end{array}$ & $\begin{array}{l}\text { Group 3: neutral } \\
(N=232)\end{array}$ & $\begin{array}{l}\text { Group 4: not } \\
\text { confident }(N=66)\end{array}$ \\
\hline \multirow[t]{9}{*}{ Socio-demographic } & Neighbourhood deprivation quintile & Reference & $p=0.06$ & $p=0.03$ & $p=0.13$ \\
\hline & First (least deprived) & $28(24.8 \%)$ & $84(22.2 \%)$ & $39(17.0 \%)$ & $9(14.1 \%)$ \\
\hline & Second & $26(23.0 \%)$ & $89(23.5 \%)$ & $41(17.8 \%)$ & $9(14.1 \%)$ \\
\hline & Third & $20(17.7 \%)$ & $71(18.8 \%)$ & $47(20.4 \%)$ & $12(18.7 \%)$ \\
\hline & Fourth & $23(20.3 \%)$ & $61(16.1 \%)$ & $50(21.7 \%)$ & $14(21.9 \%)$ \\
\hline & Fifth (most deprived) & $16(14.2 \%)$ & $73(19.3 \%)$ & $53(23.0 \%)$ & $20(31.2 \%)$ \\
\hline & Domestic status & Reference & $p=0.16$ & $p=0.01$ & $p=0.002$ \\
\hline & Married/living with partner & $87(82.1 \%)$ & $273(74.8 \%)$ & $138(63.9 \%)$ & $31(51.7 \%)$ \\
\hline & Single/widowed/divorced/separated & $19(17.9 \%)$ & $92(25.2 \%)$ & $78(36.1 \%)$ & $29(48.3 \%)$ \\
\hline \multirow{8}{*}{$\begin{array}{r}\text { Clinical and } \\
\text { treatment }\end{array}$} & Co-morbidities & Reference & $p=0.60$ & $p=0.22$ & $p<0.001$ \\
\hline & None & $42(40.0 \%)$ & $107(34.2 \%)$ & $23(12.4 \%)$ & $10(19.2 \%)$ \\
\hline & 1 & $45(42.9 \%)$ & $88(28.1 \%)$ & $61(32.8 \%)$ & $16(30.8 \%)$ \\
\hline & 2 & $13(12.4 \%)$ & $68(21.7 \%)$ & $61(32.8 \%)$ & $7(13.5 \%)$ \\
\hline & $3+$ & $5(4.8 \%)$ & $50(16.0 \%)$ & $41(22.0 \%)$ & $19(36.5 \%)$ \\
\hline & Stoma & Reference & $p=0.01$ & $p<0.001$ & $p<0.001$ \\
\hline & No & $88(76.5 \%)$ & $255(66.7 \%)$ & $130(57.3 \%)$ & $31(47.7 \%)$ \\
\hline & Yes & $27(23.5 \%)$ & $127(33.3 \%)$ & $97(42.7 \%)$ & $34(52.3 \%)$ \\
\hline \multirow[t]{4}{*}{ Symptoms } & Fatigue; mean (SD) & Reference & $p<0.001$ & $p<0.001$ & $p<0.001$ \\
\hline & & $9.3(5.0)$ & $11.9(5.0)$ & $14.8(4.8)$ & $18.9(4.7)$ \\
\hline & Pain; mean (SD) & Reference & $p=0.13$ & $p<0.001$ & $p<0.001$ \\
\hline & & $6.7(3.4)$ & $8.9(4.7)$ & $11.4(5.7)$ & $14.6(6.5)$ \\
\hline \multirow[t]{4}{*}{ Psychosocial } & Positivity; mean (SD) & Reference & $p=0.05$ & $p<0.001$ & $p<0.001$ \\
\hline & & $20.2(5.3)$ & $19.1(4.0)$ & $16.1(3.9)$ & $15.0(3.5)$ \\
\hline & Negativity; mean (SD) & Reference & $p=0.02$ & $p<0.001$ & $p<0.001$ \\
\hline & & $6.7(2.6)$ & $7.8(3.1)$ & $10.1(3.6)$ & $12.2(4.8)$ \\
\hline
\end{tabular}

Final trajectory model included all participant characteristics shown in table (i.e. those statistically significant for at least one group comparison) as well as a term indicating whether baseline questionnaire completed before or after surgery. Higher scores for fatigue and pain indicate greater problems, range 4-28 (QLACS subscales). Lower scores for positivity and greater scores for negativity indicate poorer levels of personal affect, range 5-25 (PANAS subscales). $P$ values represent significance of Wald test for each characteristic in groups 2,3 and 4, respectively, compared with group 1 (reference group). Denominators for percents vary due to missing data for some characteristics; percents are calculated out of those with data available. Extent of missing data for predictor variables: fatigue $N$ available 753, $N$ missing 3; pain $N$ available 748, $N$ missing 8; positivity $N$ available 724, $N$ missing 32; negativity $N$ available 731, $N$ missing 25 at baseline. Cut-offs used to describe categories of mean six-item SEMCD scale: 1-4 'low confidence', 5-6 'moderately confident', 7-8 'confident' and 9-10 'very confident'

$S D$ standard deviation

Exploring areas where people feel least confident to manage is important to guide specific areas for intervention. When looking at the CREW population as a whole, our results demonstrate clinically meaningful improvements in mean scores across two of the six individual items, namely, confidence to manage physical discomfort/pain and emotional distress. In contrast, self-efficacy for managing other symptoms and health-related problems showed very little change and was the area of lowest reported confidence at 2 years.
There are few comparable published studies describing domain specific confidence to manage disease-related problems. In a cross-sectional sample of 182 cancer patients within 1 year of treatment, Foster et al. [17] report similar findings with lowest levels of confidence for fatigue, emotional distress and symptoms in the first 12 months post curative intent treatment across a range of cancer types.

Due to variations in how self-efficacy is defined and measured across studies, and the discordance in populations 
and timing of assessments, there is little agreement in previous studies with regard to the pattern of self-efficacy over time with some authors reporting a decline $[10,12,33]$ and others recounting little change over a 12 -month period [5, 11]. However, none looked specifically at colorectal cancer patients, followed patients for more than 12 months nor used a comparable, psychometrically robust measure of self-efficacy, measuring confidence to manage disease-related problems.

Examination of factors associated with lower levels of self-efficacy revealed those with baseline scores indicating greater deprivation, who were single, had more co-morbidities, worse baseline fatigue or pain and had a stoma as part of their treatment (either reversed or permanent) were more likely to report lower scores. Existing literature has demonstrated an association between depression and anxiety and self-efficacy [34 12]. Whilst we found a univariate association (data not shown), these factors were not predictive of self-efficacy once other variables in the model were taken into account. Also noteworthy is the absence of influence of age or any other disease or treatment variables including site and stage of disease, and whether or not a person had received chemotherapy. These findings are similar to those reported by our group in a cross-sectional sample of cancer survivors [17]. Comparable findings have also been described in a sample of breast cancer patients undergoing adjuvant endocrine therapy. Here, Shelby et al. [9] report that cancer stage, time since surgery and receipt of chemotherapy were not significantly associated with level of selfefficacy as measured by a modified version of the 'standard efficacy scale'. This challenges the approach of stratified follow-up in cancer care which is entirely determined by disease and treatment-specific factors, with little consideration of psychosocial and symptom-related factors.

There are numerous studies describing the positive crosssectional association between self-efficacy and quality of life, adaptation to cancer diagnosis and reduced distress in cancer patients [35-38]. Strengthening the argument of the importance of self-efficacy in recovery from cancer, we reported that self-efficacy to manage disease-related problems measured at the time of diagnosis was a significant predictor of quality of life and well-being over the following 2 years in the CREW cohort [19]. Based on this evidence, we suggested that interventions to bolster self-efficacy should be offered as early as possible in order to enhance recovery. Data presented here reinforces this recommendation, as there is little evidence of spontaneous increases in self-efficacy over time. Our data indicate that problems associated with symptoms including pain, fatigue and distress are associated with the lowest self-efficacy scores and suggest important areas for intervention. The question that now needs to be addressed is how might we intervene to support those with least confidence?
Self-management support is a key component in the shift towards stratified aftercare for cancer patients in the UK and has been acknowledged internationally as an integral element to optimise health after cancer [39]. Central to selfmanagement programmes is that patients need to be equipped with the skills, knowledge and, crucially, confidence to enable effective management of the consequences of cancer and its treatment.

There has been increasing research interest in the development and testing of self-management interventions in the cancer domain with a recent systematic review identifying 42 randomised controlled trials examining self-management education [40]. A narrative qualitative synthesis revealed improvements in fatigue, pain, depression, anxiety, emotional distress and quality of life with $90 \%$ of identified studies including intervention components that facilitated self-efficacy to manage the consequences of cancer (though few measured this as an outcome). Because there was considerable heterogeneity in the intervention components, a meta-synthesis was not possible, nor agreement on the components of selfmanagement interventions associated with the greatest improvement in outcomes.

Evidence from the aforementioned review does suggest that tailoring self-management interventions to specific problems, for example, depression or distress may lead to favourable outcomes. This is in accordance with our data suggesting the importance of taking account of levels of self-efficacy for specific symptoms. This is echoed in a recently published large randomised controlled trial (RCT) evaluating a nurse-led supportive care package for colorectal cancer survivors [41]. Despite a comprehensive intervention including information, a nurse-led end of treatment session, tailored survivor care plan and telephone follow-up, the study failed to show a significance difference in their primary (distress) or secondary (survivorship care needs and quality of life) outcomes. The authors propose that this was due to the absence of tailoring of the intervention to those most in need, which they suggest should be determined by level of symptom burden. We would argue that symptom burden might not tell the whole story, and that confidence to manage, whatever the level of symptoms, may be just as important.

\section{Limitations}

Ninety-one percent of eligible patients were approached to take part in the study and response rates were high. However, some attrition is evident as would be expected in a longitudinal study with $74 \%$ of participants who were still eligible for follow-up returning questionnaire at 24 months. Those patients who declined participation in the CREW study were broadly similar to those who returned questionnaires, although there were fewer older and frail participants consented to the cohort. Those with lower baseline scores of 
self-efficacy were also less likely to return questionnaires at subsequent time points; thus, our results may underrepresent those with the lowest self-efficacy levels.

\section{Conclusion}

Our analysis has revealed four distinct groups of patients with different levels of self-efficacy. Particular attention is needed for self-management support of symptom-related problems and distress, where confidence to manage was the lowest. Clinicians can also be informed by the factors found to be associated with lower levels of self-efficacy including multico-morbidities, having a stoma and living alone, which may help them identify those patients most in need of support. Support for those reporting low confidence should be available close to the time of diagnosis, rather than waiting until the end of treatment. This would require a proactive, rather than a reactive approach to patient care. Almost $40 \%$ of participants reported suboptimal confidence to self-manage illness-related problems. Early screening and appropriate intervention could significantly increase self-efficacy and consequently enhance the quality of life and recovery experiences of this substantial group of people living with and beyond colorectal cancer. Further research is required to develop self-management interventions that consider self-efficacy to manage illnessrelated problems at diagnosis - including symptoms associated with cancer and its treatment as well as co-morbidities. They must be endorsed and supported by health care professionals and implementable within the appropriate health care system.

\begin{abstract}
Acknowledgements We thank all CREW study participants: Carol Hill, Kerry Coleman, Bjoern Schukowsky and Christine May (study administrators); Matthew Breckons, Cassandra Powers, Alex RecioSaucedo, Bina Nausheen, Ikumi Okamoto and Kim Chivers-Seymour (researchers); Jo Clough and Alison Farmer (research partners) and members of Study Advisory Committee: Jo Armes, Janis Baird, Andrew Bateman, Nick Beck, Graham Moon, Claire Hulme, Karen Poole, Susan Restorick-Banks, Paul Roderick, Claire Taylor, Jocelyn Walters, Fran Williams, Lynn Batehup, Jessica Corner and Deborah Fenlon.
\end{abstract}

Funding The CREW study is funded by Macmillan Cancer Support grant number 3546834 .

\section{Compliance with ethical standards}

Conflict of interest The authors declare that they have no conflict of interests.

Ethical approval All procedures performed in studies involving human participants were in accordance with the ethical standards of the institutional and/or national research committee and with the 1964 Helsinki Declaration and its later amendments or comparable ethical standards.

Informed consent Informed consent was obtained from all individual participants included in the study.
Open Access This article is distributed under the terms of the Creative Commons Attribution 4.0 International License (http:// creativecommons.org/licenses/by/4.0/), which permits unrestricted use, distribution, and reproduction in any medium, provided you give appropriate credit to the original author(s) and the source, provide a link to the Creative Commons license, and indicate if changes were made.

\section{References}

1. Arndt V, Merx H, Stegmaier C, Ziegler H, Brenner H. Quality of life in patients with colorectal cancer 1 year after diagnosis compared with the general population: a population-based study. J Clin Oncol. 2004;22(23):4829-36.

2. Desnoo L, Faithfull S. A qualitative study of anterior resection syndrome: the experiences of cancer survivors who have undergone resection surgery. Eur J Cancer Care. 2006;15(3):244-51.

3. Elliott J, Fallows A, Staetsky L, Smith PWF, Foster CL, Maher EJ, et al. The health and well-being of cancer survivors in the UK: findings from a population-based survey. Br J Cancer. 2011;105: S11-20.

4. Bandura A. In: Bandura A, editor. Exercise of personal and collective efficacy in changing societies. New York: Cambridge University Press; 1995.

5. Manne SL, Ostroff JS, Norton TR, Fox K, Grana G, Goldstein L. Cancer-specific self-efficacy and psychosocial and functional adaptation to early stage breast cancer. Ann Behav Med. 2006;31(2): 145-54.

6. Curtis R, Groarke A, Sullivan F. Stress and self-efficacy predict psychological adjustment at diagnosis of prostate cancer. Sci Rep. 2014;4:5569.

7. Haugland T, Wahl AK, Hofoss D, Devon HA. Association between general self-efficacy, social support, cancer-related stress and physical health-related quality of life: a path model study in patients with neuroendocrine tumors. Health Qual Life Outcomes. 2016;14

8. Zhang MF, Zheng MC, Liu WY, Wen YS, Wu XD, Liu QW. The influence of demographics, psychological factors and self-efficacy on symptom distress in colorectal cancer patients undergoing postsurgical adjuvant chemotherapy. EJON. 2015;19(1):89-96. http:// www.sciencedirect.com/science/article/pii/S1462388914001136.

9. Shelby RA, Edmond SN, Wren AA, Keefe FJ, Peppercorn JM, Marcom PK, et al. Self-efficacy for coping with symptoms moderates the relationship between physical symptoms and well-being in breast cancer survivors taking adjuvant endocrine therapy. Support Care Cancer. 2014;22(10):2851-9.

10. Lev EL, Paul D, Owen SV. Age, self-efficacy, and change in patients' adjustment to cancer. Cancer Pract. 1999;7(4):170-6.

11. Rottmann N, Dalton SO, Christensen J, Frederiksen K, Johansen C. Self-efficacy, adjustment style and well-being in breast cancer patients: a longitudinal study. Qual Life Res. 2010;19(6):827-36.

12. Mystakidou K, Tsilika E, Parpa E, Gogou P, Panagiotou I, Vassiliou I, et al. Relationship of general self-efficacy with anxiety, symptom severity and quality of life in cancer patients before and after radiotherapy treatment. Psycho-Oncology. 2013;22(5):1089-95.

13. Schwarzer RJ, Jerusalem M. In: Weinman J, Wright S, Johnson M, editors. Generalized Self-Efficacy Scale. Windsor: NFERNELSON; 1995.

14. Heitzmann CA, Merluzzi TV, Jean-Pierre P, Roscoe JA, Kirsh KL, Passik SD. Assessing self-efficacy for coping with cancer: development and psychometric analysis of the brief version of the Cancer Behavior Inventory (CBI-B). Psycho-Oncology. 2011;20(3):302-12.

15. Bandura A. Social learning theory. New York: General Learning Press; 1977. 
16. Lorig KR, Ritter P, Stewart AL, Sobel DS, Brown BW, Bandura A, et al. Chronic disease self-management program - 2-year health status and health care utilization outcomes. Med Care. 2001;39(11):1217-23.

17. Foster C, Breckons M, Cotterell P, Barbosa D, Calman L, Corner J, et al. Cancer survivors' self-efficacy to self-manage in the year following primary treatment. J Cancer Surviv. 2015;9(1):11-9.

18. Dunn J, Ng SK, Holland J, Aitken J, Youl P, Baade PD, et al. Trajectories of psychological distress after colorectal cancer. Psycho-Oncology. 2013;22(8):1759-65.

19. Foster C, Haviland J, Winter J, Grimmett C, Seymour KC, Batehup $\mathrm{L}$, et al. Pre-surgery depression and confidence to manage problems predict recovery trajectories of health and wellbeing in the first two years following colorectal cancer: results from the CREW cohort study. Plos One. 2016;11(5):e0155434.

20. Fenlon D, Richardson A, Addington-Hall J, Smith P, Corner J, Winter J, et al. A cohort study of the recovery of health and wellbeing following colorectal cancer (CREW study): protocol paper. BMC Health Serv Res. 2012;12:90.

21. Foster C, Fenlon D. Recovery and self-management support following primary cancer treatment. Br J Cancer. 2011;105:S21-S8.

22. Foster C, Breckons M, Hankins M, Fenlon D, Cotterell P. Developing a scale to measure self-efficacy to self-manage problems following cancer treatment. Psycho-Oncology. 2013;22:16.

23. Spielberger CD, Gorsuch RL, Lushene R, Vagg PR, Jacobs GA Manual for the State-Trait Anxiety Inventory (STAI). 1983. https://ubir.buffalo.edu/xmlui/handle/10477/2895.

24. Radloff S. The CES-D scale: a self-report depression scale for research in the general population. Appl Psycol Meas. 1977;1(3):385-401.

25. Katz MR, Kopek N, Waldron J, Devins GM, Tomlinson G. Screening for depression in head and neck cancer. Psychooncology. 2004;13(4): 269-80.

26. Sherbourne CD, Stewart AL. The MOS social support survey. Soc Sci Med. 1991;32(6):705-14.

27. Avis NE, Smith KW, McGraw S, Smith RG, Petronis VM, Carver CS. Assessing quality of life in adult cancer survivors (QLACS). Qual Life Res. 2005;14:1007-23.

28. Thompson ER. Development and validation of an internationally reliable short-form of the Positive and Negative Affect Schedule (PANAS). J Cross-Cult Psychol. 2007;38(2):227-42.

29. Norman GR, Sloan JA, Wyrwich KW. Interpretation of changes in health-related quality of life - the remarkable universality of half a standard deviation. Med Care. 2003;41(5):582-92.
30. Jones BL, Nagin DS, Roeder K. A SAS procedure based on mixture models for estimating developmental trajectories. Sociol Methods Res. 2001;29(3):374-93.

31. Kass RE, Raftery AE. Bayes Factors. J Am Stat Assoc. 1995;90(430):773-95. https://www.tandfonline.com/doi/abs/10. 1080/01621459.1995.1047657210.1007/s11764-017-0636-x.

32. Schwarz G. Estimating dimension of a model. Ann Stat. 1978;6(2): 461-4.

33. Paterson C, Robertson A, Nabi G. Exploring prostate cancer survivors' self-management behaviours and examining the mechanism effect that links coping and social support to health-related quality of life, anxiety and depression: a prospective longitudinal study. Eur J Oncol Nurs. 2015;19(2):120-8.

34. Mystakidou K, Parpa E, Tsilika E, Gogou P, Panagiotou I, Galanos A, et al. Self-efficacy, depression, and physical distress in males and females with cancer. Am J Hosp Palliat Med. 2010;27(8):518-25.

35. Boehmer S, Luszczynska A, Schwarzer R. Coping and quality of life after tumor surgery: personal and social resources promote different domains of quality of life. Anxiety Stress Coping. 2007;20(1):61-75.

36. Hirai K, Suzuki Y, Tsuneto S, Ikenaga M, Hosaka T, Kashiwagi T. A structural model of the relationships among self-efficacy psychological adjustment, and physical condition in Japanese advanced cancer patients. Psycho-Oncology. 2002;11(3):221-9.

37. Northouse LL, Mood D, Kershaw T, Schafenacker A, Mellon S, Walker J, et al. Quality of life of women with recurrent breast cancer and their family members. J Clin Oncol. 2002;20(19):4050-64.

38. Wang ZY, Liu L, Shi M, Wang L. Exploring correlations between positive psychological resources and symptoms of psychological distress among hematological cancer patients: a cross-sectional study. Psychol Health Med. 2016;21(5):571-82.

39. Ferrell B, McCabe MS, Levit L. The Institute of Medicine report on high-quality cancer care: implications for oncology nursing. Oncol Nurs Forum. 2013;40(6):603-9.

40. Howell D, Harth T, Brown J, Bennett C, Boyko S. Selfmanagement education interventions for patients with cancer: a systematic review. Support Care Cancer. 2017;25(4):1323-55.

41. Jefford M, Gough K, Drosdowsky A, Russell L, Aranda S, Butow $\mathrm{P}$, et al. A randomized controlled trial of a nurse-led supportive care package (SurvivorCare) for survivors of colorectal cancer. Oncologist. 2016;21(8):1014-23. 